\title{
Thermal Effect on Vibration of Tapered Rectangular Plate
}

\author{
Anupam Khanna ${ }^{1}$ and Ashish Singhal ${ }^{2}$ \\ ${ }^{1}$ Department of Mathematics, Maharishi Markandeshwar University, Ambala, Haryana 133203, India \\ ${ }^{2}$ Department of Mathematics, Northern India Engineering College, New Delhi 110053, India \\ Correspondence should be addressed to Anupam Khanna; rajieanupam@gmail.com
}

Received 23 January 2014; Revised 24 May 2014; Accepted 23 June 2014; Published 8 July 2014

Academic Editor: Lucio Nobile

Copyright (C) 2014 A. Khanna and A. Singhal. This is an open access article distributed under the Creative Commons Attribution License, which permits unrestricted use, distribution, and reproduction in any medium, provided the original work is properly cited.

\begin{abstract}
A mathematical model is constructed to help the engineers in designing various mechanical structures mostly used in satellite and aeronautical engineering. In the present model, vibration of rectangular plate with nonuniform thickness is discussed. Temperature variations are considered biparabolic, that is, parabolic in $x$-direction and parabolic in $y$-direction. The fourth-order differential equation of the motion is solved by Rayleigh Ritz method for three different boundary conditions around the boundary of plate. Numerical values of frequencies for the first two modes of vibration are presented in tabular form for different values of thermal gradient, taper constants, and aspect ratio.
\end{abstract}

\section{Introduction}

The structures are designed to support the high speed engines and turbines subjected to the vibration. Due to faulty design, there is unbalance in the engines which causes excessive and unpleasant stresses because of vibration. In the field of science and technology, it is preferred to design large machines and structures for smooth operations with controlled vibration. Recent studies in the field of vibrational behavior create a huge interest for scientists and engineers in the designs and constructions of complex systems or structures such as ships, submarines, aircrafts, launch vehicles, missiles, and satellites.

Tapered plates or plates of nonuniform thickness are commonly used in many engineering applications such as nuclear engineering, aeronautical engineering, and chemical plants, under the influence of elevated temperature to control high vibration.

A collection of research papers on vibration of plates with different shapes and boundary conditions is given by Leissa [1] in his monograph. Leissa [2] discussed different models on free vibration of rectangular plates. Jain and Soni [3] analyzed the free vibrations of rectangular plates with parabolically varying thickness. Tomar and Gupta [4] studied the effect of thermal gradient on the vibration of a rectangular plate with bidirectional variation in thickness. Leissa [5] investigated the effect of thermal gradient on the vibration of parallelogram plate with bidirectional thickness variation in both directions. Singh and Chakraverty [6] studied the transverse vibration of circular and elliptical plates with variable thickness. Sharma and Chand [7] analyzed the vibrations in transversely isotropic plates due to suddenly punched hole. Leissa [8] discussed the historical bases of the Rayleigh and Ritz methods for the vibrations of plates. Chakraverty et al. [9] studied the flexural vibrations of nonhomogeneous elliptical plates. Gupta and Khanna [10] analyzed time period and deflection for the first two modes of vibrations of viscoelastic rectangular plate with linear thickness variations in both directions. Gupta and Khanna [11] had evaluated time period and deflection for the first two modes of vibration of viscoelastic rectangular plate for biparabolic thickness variation. Lal et al. [12] analyzed the transverse vibrations of nonhomogeneous rectangular plates of uniform thickness using boundary characteristic orthogonal polynomials. Khanna et al. [13] discussed the effect of nonhomogeneity on thermally induced vibrations of nonhomogeneous rectangular plate.

In the present model, the authors analyzed vibration of rectangular plate with nonuniform thickness (Figure 1). Nonuniformity in thickness is considered biparabolic. The authors calculated frequency for the first two modes of vibration at various values of thermal gradient (due to variation in temperature), taper constant (due to nonuniform 


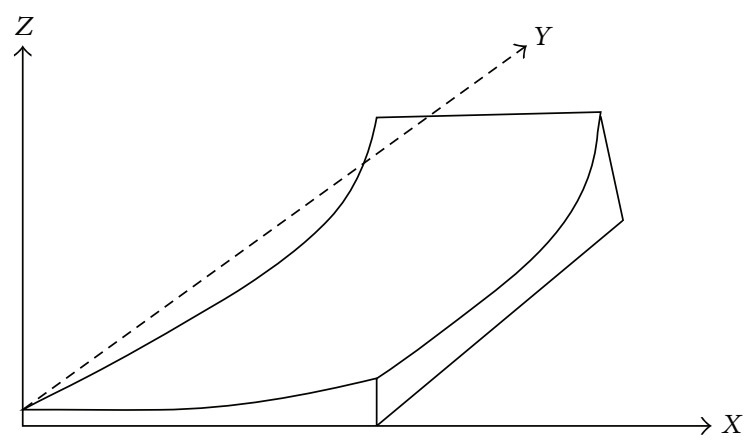

FIGURE 1: Rectangular plate with 2-directional thickness variation.

thickness), and aspect ratio (ratio of length and breadth of the plate) for three different boundary conditions, that is, $\mathrm{C}-\mathrm{C}$ C-C, S-S-S-S, and C-S-C-S where C and S stand for clamped and simply supported, respectively. Results are given in the tabular form.

\section{Materials and Methods}

2.1. Analysis of Motion. Fourth-order differential equation of motion for isotropic rectangular plate in Cartesian coordinate is [14]

$$
\begin{aligned}
& {\left[D_{1}\left(\frac{\partial^{4} W}{\partial x^{4}}+2 \frac{\partial^{4} W}{\partial x^{2} \partial y^{2}}+\frac{\partial^{4} W}{\partial y^{4}}\right)\right.} \\
& +2 \frac{\partial D_{1}}{\partial x}\left(\frac{\partial^{3} W}{\partial x^{3}}+\frac{\partial^{3} W}{\partial x \partial y^{2}}\right)+2 \frac{\partial D_{1}}{\partial y}\left(\frac{\partial^{3} W}{\partial y^{3}}+\frac{\partial^{3} W}{\partial y \partial x^{2}}\right) \\
& +\frac{\partial^{2} D_{1}}{\partial x^{2}}\left(\frac{\partial^{2} W}{\partial x^{2}}+v \frac{\partial^{2} W}{\partial y^{2}}\right)+\frac{\partial^{2} D_{1}}{\partial y^{2}}\left(\frac{\partial^{2} W}{\partial y^{2}}+v \frac{\partial^{2} W}{\partial x^{2}}\right) \\
& \left.\quad+2(1-v) \frac{\partial^{2} D_{1}}{\partial x \partial y} \frac{\partial^{2} W}{\partial x \partial y}\right]-\rho p^{2} d W=0,
\end{aligned}
$$

where $D_{1}=E d^{3} /\left(12\left(1-v^{2}\right)\right)$ is the flexural rigidity of the plate's material, $W=W(x, y)$ is the deflection function, $v$ is Poisson ratio, $\rho$ is mass per unit volume, and $d$ is thickness of the plate.

It is assumed that thickness of the rectangular plate varies parabolically in both directions [11]; that is,

$$
d=d_{0}\left(1+\beta_{1} \frac{x^{2}}{a^{2}}\right)\left(1+\beta_{2} \frac{y^{2}}{b^{2}}\right),
$$

where $a$ and $b$ are length and breadth of rectangular plate, respectively, and $\beta_{1}$ and $\beta_{2}$ are taper parameters in $x$ direction and $y$-direction, respectively.

The authors assumed biparabolic temperature variations as

$$
\tau=\tau_{0}\left(1-\frac{x^{2}}{a^{2}}\right)\left(1-\frac{y^{2}}{b^{2}}\right)
$$

where $\tau$ denotes the temperature excess above the reference temperature at any point on the plate and $\tau_{0}$ denotes the temperature excess above the reference temperature at $x=$ $y=0$.

The temperature dependence of the modulus of elasticity for most of engineering materials can be expressed as follows [15]:

$$
E=E_{0}(1-\gamma \tau)
$$

where $E_{0}$ is value of Young's modulus at reference temperature, that is, $\tau=0$, and $\gamma$ is slope of variation of $E$ and $\tau$. On using (3) in (4), one obtains

$$
E=E_{0}\left[1-\alpha\left(1-\frac{x^{2}}{a^{2}}\right)\left(1-\frac{y^{2}}{b^{2}}\right)\right],
$$

where $\alpha=\gamma \tau_{0}(0 \leq \alpha<1)$ is thermal gradient.

On substituting the values of $d$ and $E$ from (2) and (5), respectively, expression of flexural rigidity $\left(D_{1}\right)$ becomes

$$
\begin{aligned}
D_{1}= & \left(E_{0}\left[1-\alpha\left(1-\left(\frac{x^{2}}{a^{2}}\right)\right)\left(1-\left(\frac{y^{2}}{b^{2}}\right)\right)\right]\right. \\
& \left.\times d_{0}^{3}\left(1+\beta_{1}\left(\frac{x^{2}}{a^{2}}\right)\right)^{3}\left(1+\beta_{2}\left(\frac{y^{2}}{b^{2}}\right)\right)^{3}\right) \\
& \times\left(12\left(1-v^{2}\right)\right)^{-1} .
\end{aligned}
$$

\subsection{Boundary Conditions and Corresponding Deflection Func-} tion. In this model, the authors discussed three different boundary conditions, that is, C-C-C-C, S-S-S-S, and C-S-C-S around the boundary of plate.

To satisfy all these boundary conditions, deflection function $(W)$ is taken as shown in Table 1.

2.3. Solution of Frequency Equation. Rayleigh Ritz technique is applied to solve the frequency equation. In this method, one requires that the maximum strain energy $\left(S_{E}\right)$ must be equal to the maximum kinetic energy $\left(K_{E}\right)$. So it is necessary for the problem under consideration that [16]

$$
\delta\left(S_{E}-K_{E}\right)=0
$$

Now assume the nondimensional variables as

$$
X=\frac{x}{a}, \quad Y=\frac{y}{a}, \quad \bar{W}=\frac{W}{a}, \quad \bar{d}=\frac{d}{a} .
$$


TABle 1

\begin{tabular}{lc}
\hline Boundary conditions & Deflection function $(W)$ \\
\hline C-C-C-C & $W=\left[\left(\frac{x}{a}\right)^{2}\left(\frac{y}{b}\right)^{2}\left(1-\frac{x}{a}\right)^{2}\left(1-\frac{y}{b}\right)^{2}\right] \times\left[A_{1}+A_{2}\left(\frac{x}{a}\right)\left(\frac{y}{b}\right)\left(1-\frac{x}{a}\right)\left(1-\frac{y}{b}\right)\right]$ \\
\hline S-S-S-S & $W=\left[\left(\frac{x}{a}\right)\left(\frac{y}{b}\right)\left(1-\frac{x}{a}\right)\left(1-\frac{y}{b}\right)\right] \times\left[A_{1}+A_{2}\left(\frac{x}{a}\right)\left(\frac{y}{b}\right)\left(1-\frac{x}{a}\right)\left(1-\frac{y}{b}\right)\right]$ \\
\hline C-S-C-S & $W=\left[\left(\frac{x}{a}\right)^{2}\left(\frac{y}{b}\right)\left(1-\frac{x}{a}\right)^{2}\left(1-\frac{y}{b}\right)\right] \times\left[A_{1}+A_{2}\left(\frac{x}{a}\right)\left(\frac{y}{b}\right)\left(1-\frac{x}{a}\right)\left(1-\frac{y}{b}\right)\right]$ \\
\hline
\end{tabular}

TABLE 2: Frequency versus taper constants at fixed $a / b=1.5, \alpha=0.0$.

\begin{tabular}{lccccccccccc}
\hline \multirow{2}{*}{$\begin{array}{l}\text { Boundary } \\
\text { conditions }\end{array}$} & \multirow{2}{*}{$\beta_{2}$} & \multicolumn{2}{c}{0.0} & \multicolumn{2}{c}{0} & \multicolumn{2}{c}{$\beta_{1}$} & \multicolumn{2}{c}{0.6} & \multicolumn{2}{c}{0.8} \\
& & Mode I & Mode II & Mode I & Mode II & Mode I & Mode II & Mode I & Mode II & Mode I & Mode II \\
\hline C-C-C-C & \multirow{2}{*}{240.47} & 60.85 & 271.43 & 68.92 & 287.81 & 73.31 & 305.20 & 77.97 & 323.46 & 82.85 \\
S-S-S-S & 0.0 & $\mathbf{2 5 6 . 9 1}$ & $\mathbf{3 4 . 2 5}$ & $\mathbf{2 7 3 . 2 0}$ & $\mathbf{3 6 . 6 2}$ & $\mathbf{2 9 1 . 0 8}$ & $\mathbf{3 9 . 2 2}$ & $\mathbf{3 1 0 . 3 4}$ & $\mathbf{4 2 . 0 1}$ & $\mathbf{3 3 0 . 7 7}$ & $\mathbf{4 4 . 9 7}$ \\
C-S-C-S & & $\mathbf{2 8 8 . 8 2}$ & $\mathbf{4 1 . 9 4}$ & $\mathbf{3 0 5 . 1 8}$ & $\mathbf{4 4 . 9 0}$ & $\mathbf{3 2 2 . 4 7}$ & $\mathbf{4 8 . 1 6}$ & $\mathbf{3 4 0 . 5 7}$ & $\mathbf{5 1 . 6 6}$ & $\mathbf{3 5 9 . 3 7}$ & $\mathbf{5 5 . 3 9}$ \\
\hline C-C-C-C & & 277.75 & 70.26 & 294.19 & 74.65 & 311.85 & 79.36 & 330.58 & 84.35 & 350.23 & 89.56 \\
S-S-S-S & 0.2 & $\mathbf{2 8 1 . 8 9}$ & $\mathbf{3 6 . 6 3}$ & $\mathbf{2 9 9 . 5 7}$ & $\mathbf{3 9 . 1 7}$ & $\mathbf{3 1 8 . 9 5}$ & $\mathbf{4 1 . 9 7}$ & $\mathbf{3 3 9 . 7 9}$ & $\mathbf{4 4 . 9 8}$ & $\mathbf{3 6 1 . 8 7}$ & $\mathbf{4 8 . 1 7}$ \\
C-S-C-S & & $\mathbf{3 1 7 . 8 4}$ & $\mathbf{4 4 . 7 9}$ & $\mathbf{3 3 5 . 7 8}$ & $\mathbf{4 7 . 9 4}$ & $\mathbf{3 5 4 . 7 1}$ & $\mathbf{5 1 . 4 1}$ & $\mathbf{3 7 4 . 5 0}$ & $\mathbf{5 5 . 1 5}$ & $\mathbf{3 9 5 . 0 6}$ & $\mathbf{5 9 . 1 3}$ \\
\hline C-C-C-C & & 302.35 & 76.32 & 320.15 & 81.04 & 339.26 & 86.10 & 359.50 & 91.44 & 380.72 & 97.02 \\
S-S-S-S & 0.4 & $\mathbf{3 1 0 . 8 5}$ & $\mathbf{3 9 . 2 2}$ & $\mathbf{3 3 0 . 1 3}$ & $\mathbf{4 1 . 9 7}$ & $\mathbf{3 5 1 . 2 2}$ & $\mathbf{4 4 . 9 9}$ & $\mathbf{3 7 3 . 8 6}$ & $\mathbf{4 8 . 2 4}$ & $\mathbf{3 9 7 . 8 3}$ & $\mathbf{5 1 . 6 9}$ \\
C-S-C-S & & $\mathbf{3 5 1 . 6 2}$ & $\mathbf{4 7 . 8 8}$ & $\mathbf{3 7 1 . 3 8}$ & $\mathbf{5 1 . 2 5}$ & $\mathbf{3 9 2 . 2 2}$ & $\mathbf{5 4 . 9 6}$ & $\mathbf{4 1 3 . 9 9}$ & $\mathbf{5 8 . 9 6}$ & $\mathbf{4 3 6 . 5 7}$ & $\mathbf{6 3 . 2 0}$ \\
\hline C-C-C-C & & 329.48 & 82.85 & 348.78 & 87.94 & 369.47 & 93.37 & 391.37 & 99.09 & 414.32 & 105.07 \\
S-S-S-S & 0.6 & $\mathbf{3 4 2 . 9 7}$ & $\mathbf{4 2 . 0 1}$ & $\mathbf{3 6 4 . 0 4}$ & $\mathbf{4 4 . 9 7}$ & $\mathbf{3 8 7 . 0 2}$ & $\mathbf{4 8 . 2 4}$ & $\mathbf{4 1 1 . 6 5}$ & $\mathbf{5 1 . 7 6}$ & $\mathbf{4 3 7 . 7 0}$ & $\mathbf{5 5 . 4 9}$ \\
C-S-C-S & & $\mathbf{3 8 9 . 1 3}$ & $\mathbf{5 1 . 1 9}$ & $\mathbf{4 1 0 . 9 3}$ & $\mathbf{5 4 . 8 0}$ & $\mathbf{4 3 3 . 8 8}$ & $\mathbf{5 8 . 7 6}$ & $\mathbf{4 5 7 . 8 4}$ & $\mathbf{6 3 . 0 4}$ & $\mathbf{4 8 2 . 6 7}$ & $\mathbf{6 7 . 5 7}$ \\
\hline C-C-C-C & & 358.70 & 89.75 & 379.61 & 95.21 & 402.00 & 101.04 & 425.68 & 107.17 & 450.47 & 113.58 \\
S-S-S-S & 0.8 & $\mathbf{3 7 7 . 6 1}$ & $\mathbf{4 4 . 9 6}$ & $\mathbf{4 0 0 . 5 9}$ & $\mathbf{4 8 . 1 5}$ & $\mathbf{4 2 5 . 6 1}$ & $\mathbf{5 1 . 6 8}$ & $\mathbf{4 5 2 . 4 0}$ & $\mathbf{5 5 . 4 8}$ & $\mathbf{4 8 0 . 6 9}$ & $\mathbf{5 9 . 5 1}$ \\
C-S-C-S & & $\mathbf{4 2 9 . 5 6}$ & $\mathbf{5 4 . 6 8}$ & $\mathbf{4 5 3 . 5 4}$ & $\mathbf{5 8 . 5 3}$ & $\mathbf{4 7 8 . 7 7}$ & $\mathbf{6 2 . 7 7}$ & $\mathbf{5 0 5 . 0 9}$ & $\mathbf{6 7 . 3 3}$ & $\mathbf{5 3 2 . 3 6}$ & $\mathbf{7 2 . 1 8}$ \\
\hline
\end{tabular}

The expressions for kinetic energy $\left(K_{E}\right)$ and strain energy $\left(S_{E}\right)$ are

$$
\begin{aligned}
& K_{E}= \frac{1}{2} \rho p^{2} \overline{d_{0}} a^{5} \\
& \times \int_{0}^{1} \int_{0}^{b / a}\left[\left(1+\beta_{1} X^{2}\right)\left(1+\beta_{2} \frac{a^{2}}{b^{2}} Y^{2}\right) \bar{W}^{2}\right] d Y d X \\
& S_{E}= \frac{E_{0} \bar{d}_{0}^{3} a^{3}}{24\left(1-v^{2}\right)} \\
& \times \int_{0}^{1} \int_{0}^{b / a}\left\{1-\alpha\left(1-X^{2}\right)\left(1-\frac{a^{2}}{b^{2}} Y^{2}\right)\right\} \\
& \quad \times\left(1+\beta_{1} X^{2}\right)^{3}\left(1+\beta_{2} \frac{a^{2}}{b^{2}} Y^{2}\right)^{3} \\
& \quad\left[\left(\frac{\partial^{2} \bar{W}}{\partial X^{2}}\right)^{2}+\left(\frac{\partial^{2} \bar{W}}{\partial Y^{2}}\right)^{2}+2 v \frac{\partial^{2} \bar{W}}{\partial X^{2}} \frac{\partial^{2} \bar{W}}{\partial Y^{2}}\right. \\
&\left.\quad+2(1-v)\left(\frac{\partial^{2} \bar{W}}{\partial X \partial Y}\right)^{2}\right] d Y d X
\end{aligned}
$$

On using (9) in (7), one gets

$$
S_{E}^{*}-\Omega^{2} K_{E}^{*}=0,
$$

where

$$
\begin{gathered}
K_{E}^{*}=\int_{0}^{1} \int_{0}^{b / a}\left[\left(1+\beta_{1} X^{2}\right)\left(1+\beta_{2} \frac{a^{2}}{b^{2}} Y^{2}\right) \bar{W}^{2}\right] d Y d X \\
S_{E}^{*}=\int_{0}^{1} \int_{0}^{b / a}\left\{1-\alpha\left(1-X^{2}\right)\left(1-\frac{a^{2}}{b^{2}} Y^{2}\right)\right\} \\
\times\left(1+\beta_{1} X^{2}\right)^{3}\left(1+\beta_{2} \frac{a^{2}}{b^{2}} Y^{2}\right)^{3} \\
\times\left[\left(\frac{\partial^{2} \bar{W}}{\partial X^{2}}\right)^{2}+\left(\frac{\partial^{2} \bar{W}}{\partial Y^{2}}\right)^{2}+2 v \frac{\partial^{2} \bar{W}}{\partial X^{2}} \frac{\partial^{2} \bar{W}}{\partial Y^{2}}\right. \\
\left.+2(1-v)\left(\frac{\partial^{2} \bar{W}}{\partial X \partial Y}\right)^{2}\right] d Y d X
\end{gathered}
$$

Here, $\Omega^{2}=\left(12 \rho p^{2} a^{2}\left(1-v^{2}\right)\right) /\left(E_{0}{\overline{d_{0}}}^{2}\right)$ is a frequency parameter. 
TABLE 3: Frequency versus taper constants at fixed $a / b=1.5, \alpha=0.2$.

\begin{tabular}{|c|c|c|c|c|c|c|c|c|c|c|c|}
\hline \multirow{3}{*}{$\begin{array}{l}\text { Boundary } \\
\text { conditions }\end{array}$} & \multirow{3}{*}{$\beta_{2}$} & \multicolumn{10}{|c|}{$\beta_{1}$} \\
\hline & & \multicolumn{2}{|c|}{0} & \multicolumn{2}{|c|}{0.2} & \multicolumn{2}{|c|}{0.4} & \multicolumn{2}{|c|}{0.6} & \multicolumn{2}{|c|}{0.8} \\
\hline & & Mode I & Mode II & Mode I & Mode II & Mode I & Mode II & Mode I & Mode II & Mode I & Mode II \\
\hline C-C-C-C & & 244.24 & 61.78 & 259.23 & 65.80 & 275.35 & 70.12 & 292.47 & 74.69 & 310.43 & 79.47 \\
\hline S-S-S-S & 0 & 246.02 & 32.58 & 262.23 & 34.93 & 280.01 & 37.51 & 299.14 & 40.27 & 319.4 & 43.19 \\
\hline C-S-C-S & & 276.34 & 39.90 & 292.34 & 42.84 & 309.24 & 46.07 & 326.93 & 49.55 & 345.32 & 53.24 \\
\hline C-C-C-C & & 266.18 & 67.25 & 282.38 & 71.57 & 299.79 & 76.20 & 318.24 & 81.08 & 337.61 & 86.19 \\
\hline S-S-S-S & 0.2 & 271.74 & 34.94 & 289.35 & 37.47 & 308.63 & 40.24 & 329.34 & 43.22 & 351.27 & 46.37 \\
\hline C-S-C-S & & 306.35 & 42.71 & 323.95 & 45.85 & 342.53 & 49.30 & 361.95 & 53.01 & 382.12 & 56.94 \\
\hline C-C-C-C & & 291.10 & 73.32 & 308.67 & 77.96 & 327.53 & 82.92 & 347.50 & 88.16 & 368.44 & 93.63 \\
\hline S-S-S-S & 0.4 & 301.30 & 37.51 & 320.52 & 40.24 & 341.51 & 43.23 & 364.03 & 46.46 & 387.85 & 49.87 \\
\hline C-S-C-S & & 340.94 & 45.77 & 360.39 & 49.13 & 380.89 & 52.81 & 402.31 & 56.78 & 424.53 & 60.98 \\
\hline C-C-C-C & & 318.49 & 79.81 & 337.56 & 84.81 & 358.00 & 90.14 & 379.63 & 95.76 & 402.30 & 101.62 \\
\hline S-S-S-S & 0.6 & 333.89 & 40.27 & 354.89 & 43.21 & 377.77 & 46.45 & 402.29 & 49.94 & 428.19 & 53.63 \\
\hline C-S-C-S & & 379.07 & 49.04 & 400.57 & 52.63 & 423.20 & 56.56 & 446.83 & 60.80 & 471.322 & 65.30 \\
\hline C-C-C-C & & 347.88 & 86.65 & 368.57 & 92.02 & 390.71 & 97.74 & 414.12 & 103.77 & 438.64 & 110.04 \\
\hline S-S-S-S & 0.8 & 368.87 & 43.18 & 391.78 & 46.35 & 416.71 & 49.85 & 443.36 & 53.62 & 471.51 & 57.62 \\
\hline C-S-C-S & & 419.95 & 52.47 & 443.65 & 56.30 & 468.57 & 60.51 & 494.57 & 65.05 & 521.51 & 69.85 \\
\hline
\end{tabular}

TABLE 4: Frequency versus taper constants at fixed $a / b=1.5, \alpha=0.6$.

\begin{tabular}{|c|c|c|c|c|c|c|c|c|c|c|c|}
\hline \multirow[t]{2}{*}{$\begin{array}{l}\text { Boundary } \\
\text { conditions }\end{array}$} & \multirow[t]{2}{*}{$\beta_{2}$} & \multicolumn{10}{|c|}{$\begin{array}{l}\beta_{1} \\
0.4\end{array}$} \\
\hline & & Mode I & Mode II & Mode I & Mode II & Mode I & Mode II & Mode I & Mode II & Mode I & Mode II \\
\hline C-C-C-C & \multirow{3}{*}{0} & 218.37 & 55.16 & 232.93 & 59.04 & 248.58 & 63.19 & 265.19 & 67.57 & 282.61 & 72.14 \\
\hline S-S-S-S & & 222.63 & 28.95 & 238.78 & 31.28 & 256.45 & 33.82 & 275.40 & 36.53 & 295.43 & 39.39 \\
\hline C-S-C-S & & 249.51 & 35.45 & 264.78 & 38.37 & 280.92 & 41.58 & 297.81 & 45.01 & 315.35 & 48.64 \\
\hline C-C-C-C & \multirow{3}{*}{0.2} & 241.39 & 60.73 & 257.16 & 64.89 & 274.10 & 69.33 & 292.05 & 74.02 & 310.88 & 78.90 \\
\hline S-S-S-S & & 250.19 & 31.28 & 267.73 & 33.78 & 286.88 & 36.52 & 307.39 & 39.45 & 329.06 & 42.53 \\
\hline C-S-C-S & & 281.98 & 38.22 & 298.91 & 41.34 & 316.77 & 44.75 & 335.44 & 48.42 & 354.82 & 52.28 \\
\hline C-C-C-C & \multirow{3}{*}{0.4} & 267.22 & 66.80 & 284.36 & 71.27 & 302.74 & 76.04 & 322.21 & 81.06 & 342.61 & 86.29 \\
\hline S-S-S-S & & 281.23 & 33.81 & 300.37 & 36.51 & 321.21 & 39.47 & 343.51 & 42.64 & 367.06 & 45.98 \\
\hline C-S-C-S & & 318.52 & 41.21 & 337.33 & 44.54 & 357.16 & 48.19 & 377.88 & 52.11 & 399.36 & 56.24 \\
\hline C-C-C-C & \multirow{3}{*}{0.6} & 295.31 & 73.22 & 313.95 & 78.03 & 333.92 & 83.16 & 355.05 & 88.55 & 377.17 & 94.16 \\
\hline S-S-S-S & & 314.95 & 36.52 & 335.85 & 39.43 & 358.57 & 42.63 & 382.86 & 46.06 & 408.48 & 49.69 \\
\hline C-S-C-S & & 358.12 & 44.40 & 379.01 & 47.96 & 401.01 & 51.86 & 423.96 & 56.04 & 447.75 & 60.47 \\
\hline C-C-C-C & \multirow{3}{*}{0.8} & 325.22 & 79.92 & 345.47 & 85.10 & 367.14 & 90.60 & 390.04 & 96.38 & 414.01 & 102.40 \\
\hline S-S-S-S & & 350.74 & 39.36 & 373.54 & 42.51 & 398.30 & 45.97 & 424.72 & 49.68 & 452.58 & 53.62 \\
\hline C-S-C-S & & 400.05 & 47.74 & 423.17 & 51.54 & 447.48 & 55.71 & 472.83 & 60.18 & 499.09 & 64.92 \\
\hline
\end{tabular}

Equation (10) consists of two unknown constants, that is, $A_{1}$ and $A_{2}$, arising due to the substitution of $W$. These two constants are to be determined as follows:

$$
\frac{\partial}{\partial A_{n}}\left(S_{E}^{*}-\Omega^{2} K_{E}^{*}\right)=0, \quad n=1,2 .
$$

On simplifying (12), one gets

$$
b_{n 1} A_{1}+b_{n 2} A_{2}=0, \quad n=1,2,
$$

where $b_{n 1}$ and $b_{n 2}$ include parametric constant, that is, taper constants, thermal gradient, aspect ratio, and frequency parameter.

For a nontrivial solution, determinant of the coefficient must be zero. So, one gets the frequency equation as

$$
\left|\begin{array}{ll}
b_{11} & b_{12} \\
b_{21} & b_{22}
\end{array}\right|=0
$$


TABLE 5: Frequency versus aspect ratio.

\begin{tabular}{lccccccccccc}
\hline \multirow{2}{*}{$\begin{array}{l}\text { Boundary } \\
\text { conditions }\end{array}$} & \multirow{2}{*}{$a / b$} & \multicolumn{2}{c}{$\alpha=\beta_{1}=\beta_{2}=0.0$} & \multicolumn{2}{c}{$\alpha=\beta_{1}=\beta_{2}=0.2$} & \multicolumn{2}{c}{$\alpha=\beta_{1}=\beta_{2}=0.4$} & \multicolumn{2}{c}{$\alpha=\beta_{1}=\beta_{2}=0.6$} & \multicolumn{2}{c}{$\alpha=\beta_{1}=\beta_{2}=0.8$} \\
& & Mode I & Mode II & Mode I & Mode II & Mode I & Mode II & Mode I & Mode II & Mode I & Mode II \\
\hline C-C-C-C & & 104.94 & 26.24 & 115.80 & 28.96 & 129.50 & 32.20 & 145.99 & 35.82 & 165.16 & 39.72 \\
S-S-S-S & 0.5 & $\mathbf{1 0 5 . 9 1}$ & $\mathbf{1 3 . 1 7}$ & $\mathbf{1 1 9 . 2 9}$ & $\mathbf{1 4 . 3 2}$ & $\mathbf{1 3 6 . 6 7}$ & $\mathbf{1 5 . 6 9}$ & $\mathbf{1 5 7 . 8 2}$ & $\mathbf{1 7 . 2 9}$ & $\mathbf{1 8 2 . 4 8}$ & $\mathbf{1 9 . 1 3}$ \\
C-S-C-S & & $\mathbf{8 6 . 9 1}$ & $\mathbf{2 5 . 3 8}$ & $\mathbf{9 6 . 3 6}$ & $\mathbf{2 8 . 1 3}$ & $\mathbf{1 0 8 . 4 9}$ & 31.44 & $\mathbf{1 2 3 . 2 9}$ & $\mathbf{3 5 . 2 1}$ & $\mathbf{1 4 0 . 6 6}$ & $\mathbf{3 9 . 3 4}$ \\
\hline C-C-C-C & & 150.10 & 38.35 & 165.27 & 42.35 & 184.37 & 47.10 & 207.31 & 52.41 & 233.97 & 58.14 \\
S-S-S-S & 1 & $\mathbf{1 4 9 . 6 7}$ & $\mathbf{2 1 . 0 8}$ & $\mathbf{1 6 8 . 5 5}$ & $\mathbf{2 3 . 1 5}$ & $\mathbf{1 9 3 . 1 1}$ & $\mathbf{2 5 . 7 2}$ & $\mathbf{2 2 3 . 0 6}$ & $\mathbf{2 8 . 8 0}$ & $\mathbf{2 5 8 . 0 6}$ & $\mathbf{3 2 . 3 9}$ \\
C-S-C-S & & $\mathbf{1 5 1 . 3 6}$ & $\mathbf{3 0 . 9 5}$ & $\mathbf{1 6 9 . 1 1}$ & $\mathbf{3 4 . 1 4}$ & $\mathbf{1 9 1 . 9 5}$ & $\mathbf{3 8 . 0 4}$ & $\mathbf{2 1 9 . 6 4}$ & $\mathbf{4 2 . 5 8}$ & $\mathbf{2 5 1 . 8 8}$ & $\mathbf{4 7 . 7 4}$ \\
\hline C-C-C-C & & 256.20 & 60.85 & 282.38 & 71.57 & 315.37 & 79.58 & 355.05 & 88.55 & 401.16 & 98.21 \\
S-S-S-S & 1.5 & $\mathbf{2 5 6 . 9 1}$ & $\mathbf{3 4 . 2 5}$ & $\mathbf{2 8 9 . 3 5}$ & $\mathbf{3 7 . 4 7}$ & $\mathbf{3 3 1 . 5 1}$ & $\mathbf{4 1 . 4 0}$ & $\mathbf{3 8 2 . 8 6}$ & $\mathbf{4 6 . 0 6}$ & $\mathbf{4 4 2 . 8 2}$ & $\mathbf{5 1 . 4 9}$ \\
C-S-C-S & & $\mathbf{2 8 8 . 8 2}$ & $\mathbf{4 1 . 9 4}$ & $\mathbf{3 2 3 . 9 5}$ & $\mathbf{4 5 . 8 5}$ & $\mathbf{3 6 9 . 2 2}$ & $\mathbf{5 0 . 5 6}$ & $\mathbf{4 2 3 . 9 6}$ & $\mathbf{5 6 . 0 4}$ & $\mathbf{4 8 7 . 5 0}$ & $\mathbf{6 2 . 2 8}$ \\
\hline C-C-C-C & & 419.78 & 104.96 & 463.20 & 115.86 & 518.02 & 128.80 & 583.98 & 143.29 & 660.66 & 158.90 \\
S-S-S-S & 2 & $\mathbf{4 2 3 . 6 4}$ & $\mathbf{5 2 . 7 1}$ & $\mathbf{4 7 7 . 1 7}$ & $\mathbf{5 7 . 2 9}$ & $\mathbf{5 4 6 . 7 1}$ & $\mathbf{6 2 . 7 8}$ & $\mathbf{6 3 1 . 2 9}$ & $\mathbf{6 9 . 1 9}$ & $\mathbf{7 2 9 . 9 3}$ & $\mathbf{7 6 . 5 5}$ \\
C-S-C-S & & $\mathbf{4 9 1 . 3 6}$ & $\mathbf{5 8 . 8 4}$ & $\mathbf{5 5 1 . 8 7}$ & $\mathbf{6 3 . 7 8}$ & $\mathbf{6 2 9 . 8 5}$ & $\mathbf{6 9 . 5 8}$ & $\mathbf{7 2 4 . 1 1}$ & $\mathbf{7 6 . 2 2}$ & $\mathbf{8 3 3 . 3 7}$ & $\mathbf{8 3 . 6 7}$ \\
\hline C-C-C-C & & 636.02 & 157.78 & 702.29 & 174.16 & 786.05 & 193.58 & 886.90 & 215.34 & 1004.13 & 238.79 \\
S-S-S-S & 2.5 & $\mathbf{6 4 4 . 3 8}$ & $\mathbf{7 6 . 4 4}$ & $\mathbf{7 2 5 . 8 4}$ & $\mathbf{8 2 . 6 6}$ & $\mathbf{8 3 1 . 6 1}$ & $\mathbf{8 9 . 9 8}$ & $\mathbf{9 6 0 . 1 9}$ & $\mathbf{9 8 . 3 8}$ & $\mathbf{1 1 1 0 . 0 2}$ & $\mathbf{1 0 7 . 8 8}$ \\
C-S-C-S & & $\mathbf{7 5 5 . 1 6}$ & $\mathbf{8 1 . 5 5}$ & $\mathbf{8 4 8 . 6 3}$ & $\mathbf{8 7 . 8 6}$ & $\mathbf{9 6 9 . 1 0}$ & $\mathbf{9 5 . 0 7}$ & $\mathbf{1 1 1 4 . 6 7}$ & $\mathbf{1 0 3 . 1 4 6}$ & $\mathbf{1 2 8 3 . 3 2}$ & $\mathbf{1 1 2 . 0 3}$ \\
\hline
\end{tabular}

With the help of (14), one can obtain a quadratic equation in $\Omega^{2}$ from which the two values of frequency parameter for both the modes of vibration can be evaluated easily.

\section{Results and Discussion}

Computations have been made for calculating frequencies for different values of thermal gradient $(\alpha)$, taper constants $\left(\beta_{1}\right.$ and $\left.\beta_{2}\right)$, and aspect ratio $(a / b)$ for the first two modes of vibration. In calculation, Poisson ratio is considered as constant, that is, 0.345 , and thickness of the plate at $X=Y=0$ is taken as $0.01 \mathrm{~m}$. In Tables 2, 3, and 4, frequency for the first two modes of vibration is reported for various values of taper constant $\left(\beta_{1}\right.$ and $\left.\beta_{2}\right)$ and thermal gradient $(\alpha)$ at fixed aspect ratio $(a / b=1.5)$. Also, frequency is tabulated for various values of aspect ratio in Table 5 for the following cases: (i) $\alpha$ $=\beta_{1}=\beta_{2}=0.0$, (ii) $\alpha=\beta_{1}=\beta_{2}=0.2$, (iii) $\alpha=\beta_{1}=\beta_{2}=0.4$, (iv) $\alpha=\beta_{1}=\beta_{2}=0.6$, and (v) $\alpha=\beta_{1}=\beta_{2}=0.8$.

In Tables $2-4$, the authors found that frequency for both the modes of vibration continuously increases as taper constants $\left(\beta_{1}\right.$ and $\left.\beta_{2}\right)$ increase from 0.0 to 0.8 for increasing values of thermal gradient $(\alpha)$.

It is interesting to notice that the first mode of frequency is maximum at C-S-C-S boundary condition while it is minimum at C-C-C-C boundary condition. But for the second mode of frequency, it is vice versa; that is, the second mode is maximum at C-C-C-C boundary condition while it is minimum at S-S-S-S boundary condition.

Here, the authors also noticed that as thermal gradient $(\alpha)$ increases from 0.0 (Table 2) to 0.6 (Table 4), frequency for both the modes decreases at all values of taper parameters for these boundary conditions.

From Table 5, the authors found that frequency increases for both the modes of vibration for these boundary conditions as aspect ratio increases from 0.5 to 2.5 . Frequency also
TABLE 6: Frequency versus thermal gradient* .

\begin{tabular}{lcccc}
\hline \multirow{2}{*}{$\alpha$} & \multicolumn{2}{c}{$\beta_{1}=0.0, \beta_{2}=0.0$} & \multicolumn{2}{c}{$\beta_{1}=0.2, \beta_{2}=0.6$} \\
& Mode I & Mode II & Mode I & Mode II \\
\hline \multirow{2}{*}{0.0} & 240.47 & 60.84 & 340.87 & 85.87 \\
& $\mathbf{2 4 0 . 4 7}$ & $\mathbf{6 4 . 8 4}$ & $\mathbf{3 4 8 . 7 8}$ & $\mathbf{8 7 . 9 4}$ \\
\hline \multirow{2}{*}{0.2} & 228.13 & 57.72 & 322.11 & 81.42 \\
& $\mathbf{2 4 4 . 2 4}$ & $\mathbf{6 1 . 7 8}$ & $\mathbf{3 3 7 . 5 6}$ & $\mathbf{8 4 . 8 1}$ \\
\hline \multirow{2}{*}{0.4} & 215.08 & 54.42 & 305.99 & 76.88 \\
& $\mathbf{2 3 1 . 6 7}$ & $\mathbf{5 8 . 5 7}$ & $\mathbf{3 2 5 . 9 6}$ & $\mathbf{8 1 . 5 2}$ \\
\hline \multirow{2}{*}{0.6} & 201.97 & 51.06 & 284.00 & 71.90 \\
& $\mathbf{2 1 8 . 3 7}$ & $\mathbf{5 5 . 1 6}$ & $\mathbf{3 1 3 . 9 5}$ & $\mathbf{7 8 . 0 3}$ \\
\hline \multirow{2}{*}{0.8} & 186.26 & 47.13 & 265.05 & 66.19 \\
& $\mathbf{2 0 4 . 2 2}$ & $\mathbf{5 1 . 5 0}$ & $\mathbf{3 0 1 . 7 4}$ & $\mathbf{7 4 . 3 0}$ \\
\hline
\end{tabular}

${ }^{*}$ Results of the present paper are shown in bold text.

increases as combined values of $\alpha, \beta_{1}$ and $\beta_{2}$ increase from 0.0 to 0.8 .

\section{Comparison}

In Tables 6 and 7, frequency for both the modes of the present paper is compared with [17] for various values of taper constants, thermal gradient, and aspect ratio.

Table 6 shows values of frequency for the first two modes of vibration with increasing values of thermal gradient for fixed values of taper constants. Here, the authors noticed that both the modes of frequency in the present paper are greater than [17] at corresponding values of thermal gradient and taper parameters.

Thickness variation in present paper is taken as biparabolic while, in [17], it is linear in $x$-direction and parabolic in $y$-direction. Therefore, the authors compared 
TABLE 7: Frequency versus taper constant $\left(\beta_{1}\right)^{*}$.

\begin{tabular}{lcc}
\hline$\beta_{1}$ & & $\alpha=0.0, \beta_{2}=0.0$ \\
& Mode I & Mode II \\
\hline \multirow{2}{*}{0.0} & 240.47 & 60.84 \\
& $\mathbf{2 4 0 . 4 7}$ & $\mathbf{6 0 . 8 4}$ \\
\hline \multirow{2}{*}{0.2} & 261.60 & 66.34 \\
& $\mathbf{2 7 1 . 4 3}$ & $\mathbf{6 8 . 9 2}$ \\
\hline \multirow{2}{*}{0.4} & 282.61 & 72.59 \\
& $\mathbf{2 8 7 . 8 1}$ & $\mathbf{7 3 . 3 1}$ \\
\hline \multirow{2}{*}{0.6} & 301.10 & 78.00 \\
& $\mathbf{3 0 5 . 2 0}$ & $\mathbf{7 7 . 9 7}$ \\
\hline \multirow{2}{*}{0.8} & 319.89 & 84.00 \\
& $\mathbf{3 2 3 . 4 6}$ & $\mathbf{8 2 . 8 5}$ \\
\hline
\end{tabular}

${ }^{*}$ Results of the present paper are shown in bold text.

both the modes of frequency of present paper with [17] for increasing values of $\beta_{1}$ only at $\alpha=0.0, \beta_{2}=0.0$ as shown in Table 7.

Here, the authors found that the first mode of frequency is greater than [17] and it is continuously increasing with increasing values of $\beta_{1}$ but the second mode of frequency is greater than [17] for $\beta_{1}=0.0,0.2,0.4$, and at $\beta_{1}=0.6,0.8$, it is smaller than [17].

\section{Conclusions}

On behalf of the above comparisons, the authors conclude the following.

(i) Frequency in the present paper (biparabolic temperature variation) is greater than [17] (linear temperature variation).

(ii) The first mode of frequency in the present paper (biparabolic thickness variation) is greater than [17] (bidimensional thickness variation, that is, linear in $x$-direction and parabolic in $y$-direction).

(iii) The main emphasis of the present study is to provide some numerical data about the first few modes of vibration for design engineers and researchers to fulfill their requirements. The authors suggest that the readers and engineers can obtain the desired values of frequency by appropriate tapering of the plate and can provide more realistic and reliable structures and designs.

\section{Conflict of Interests}

The authors declare that there is no conflict of interests regarding the publication of this paper.

\section{References}

[1] A. W. Leissa, "Vibration of plates," Tech. Rep. SP-160, NASA, 1969.

[2] A. W. Leissa, "The free vibration of rectangular plates," Journal of Sound and Vibration, vol. 31, no. 3, pp. 257-293, 1973.
[3] R. K. Jain and S. R. Soni, "Free vibrations of rectangular plates of parabolically varying thickness," Indian Journal of Pure and Applied Mathematics, vol. 4, no. 3, pp. 267-277, 1973.

[4] J. S. Tomar and A. K. Gupta, "Effect of thermal gradient on frequencies of an orthotropic rectangular plate whose thickness varies in two directions," Journal of Sound and Vibration, vol. 98, no. 2, pp. 257-262, 1985.

[5] A. W. Leissa, "Recent studies in plate vibration 1981-1985 part II: complicating effects," Shock and Vibration Digest, vol. 19, no. 3, pp. 10-24, 1987.

[6] B. Singh and S. Chakraverty, "Transverse vibration of circular and elliptical plates with variable thickness," Indian Journal of Pure Applied Mathematics, vol. 22, no. 9, pp. 787-803, 1991.

[7] J. N. Sharma and D. Chand, "Vibrations in a transversely isotropic plate due to suddenly punched hole," Indian Journal of Pure and Applied Mathematics, vol. 27, no. 2, pp. 217-226, 1996.

[8] A. W. Leissa, "The historical bases of the Rayleigh and Ritz methods," Journal of Sound and Vibration, vol. 287, no. 4-5, pp. 961-978, 2005.

[9] S. Chakraverty, R. Jindal, and V. K. Agarwal, "Flexural vibrations of non-homogeneous elliptic plates," Indian Journal of Engineering and Materials Sciences, vol. 12, no. 6, pp. 521-528, 2005.

[10] A. K. Gupta and A. Khanna, "Vibration of visco-elastic rectangular plate with linearly thickness variations in both directions," Journal of Sound and Vibration, vol. 301, no. 3-5, pp. 450-457, 2007.

[11] A. K. Gupta and A. Khanna, "Vibration of clamped visco-elastic rectangular plate with parabolic thickness variations," Shock and Vibration, vol. 15, no. 6, pp. 713-723, 2008.

[12] R. Lal, Y. Kumar, and U. S. Gupta, “Transverse vibrations of nonhomogeneous rectangular plates of uniform thickness using boundary characteristic orthogonal polynomials," International Journal of Applied Mathematics and Mechanics, vol. 6, no. 14, pp. 93-109, 2009.

[13] A. Khanna, N. Kaur, and A. K. Sharma, "Effect of varying poisson ratio on thermally induced vibrations of non-homogeneous rectangular plate," Indian Journal of Science and Technology, vol. 5, no. 9, pp. 3263-3267, 2012.

[14] A. K. Gupta and L. Kumar, "Thermal effect on vibration of nonhomogenous visco-elastic rectangular plate of linearly varying thickness," Meccanica, vol. 43, no. 1, pp. 47-54, 2008.

[15] A. Khanna and A. K. Sharma, "Natural vibration of viscoelastic plate of varying thickness with thermal effect," Journal of Applied Science and Engineering, vol. 16, no. 2, pp. 135-140, 2013.

[16] A. Khanna and P. Arora, "Effect of sinusoidal thickness variation on vibrations of non-homogeneous parallelogram plate with bi-linearly temperature variations," Indian Journal of Science and Technology, vol. 6, no. 9, pp. 5228-5234, 2013.

[17] A. Kumar Gupta, V. Panwar, and R. P. Vats, "Vibrations of nonhomogeneous rectangular plate of variable thickness in both directions with thermal gradient effect," International Journal of Applied Mathematics and Mechanics, vol. 6, no. 16, pp. 19-37, 2010. 

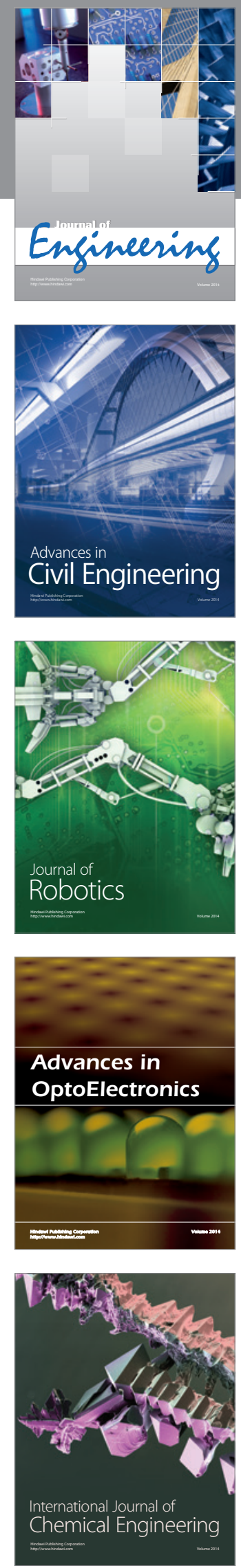

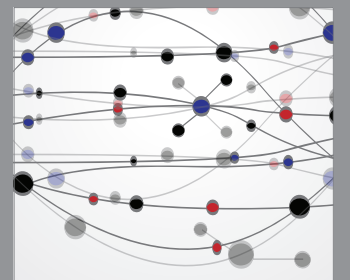

The Scientific World Journal
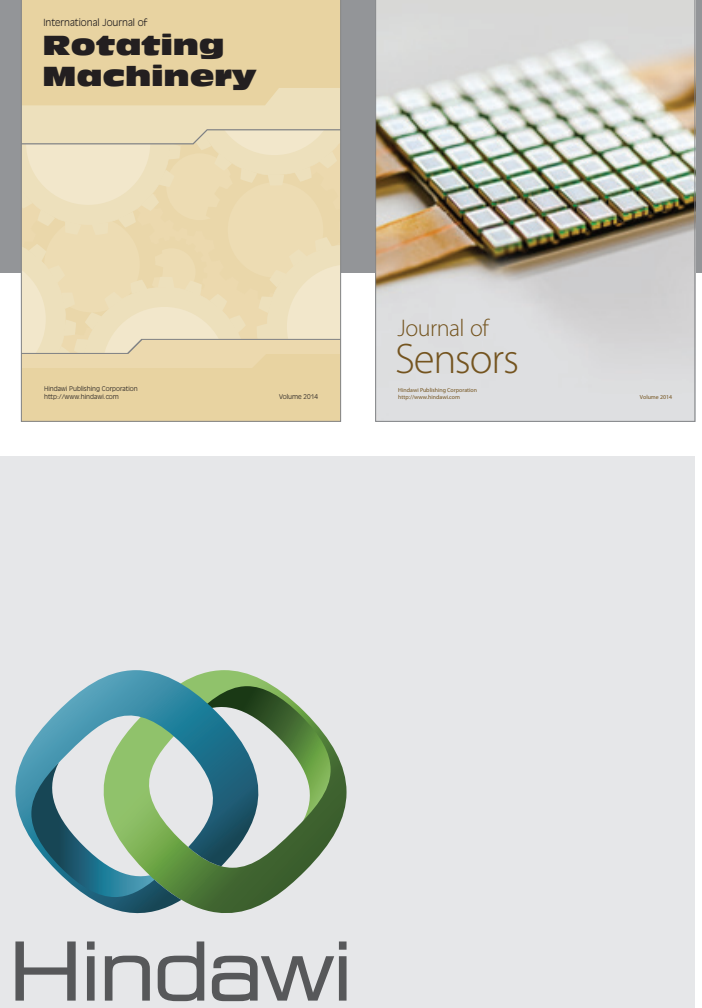

Submit your manuscripts at http://www.hindawi.com
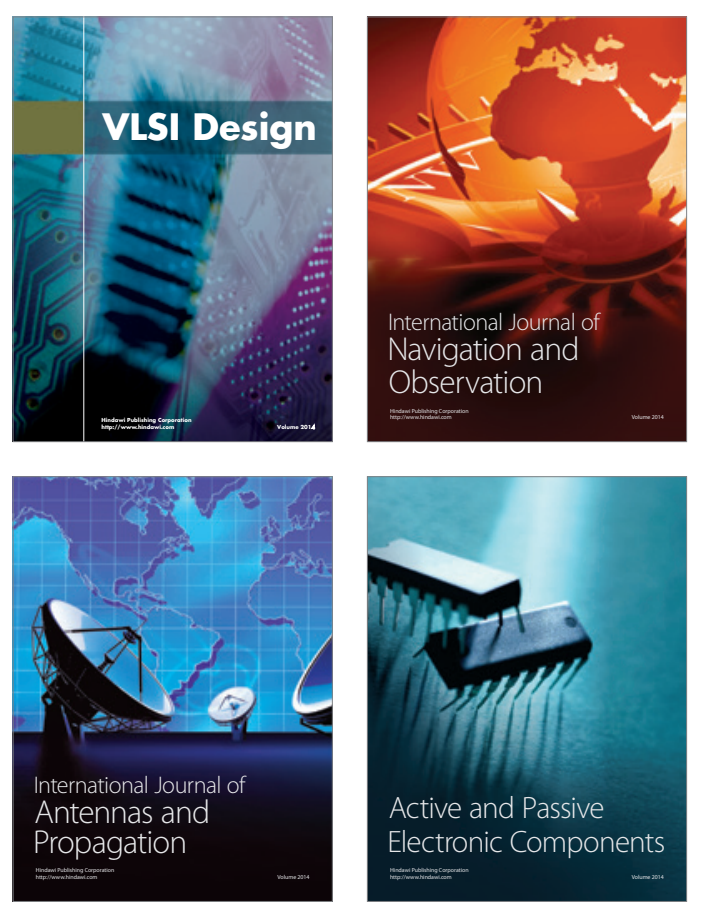
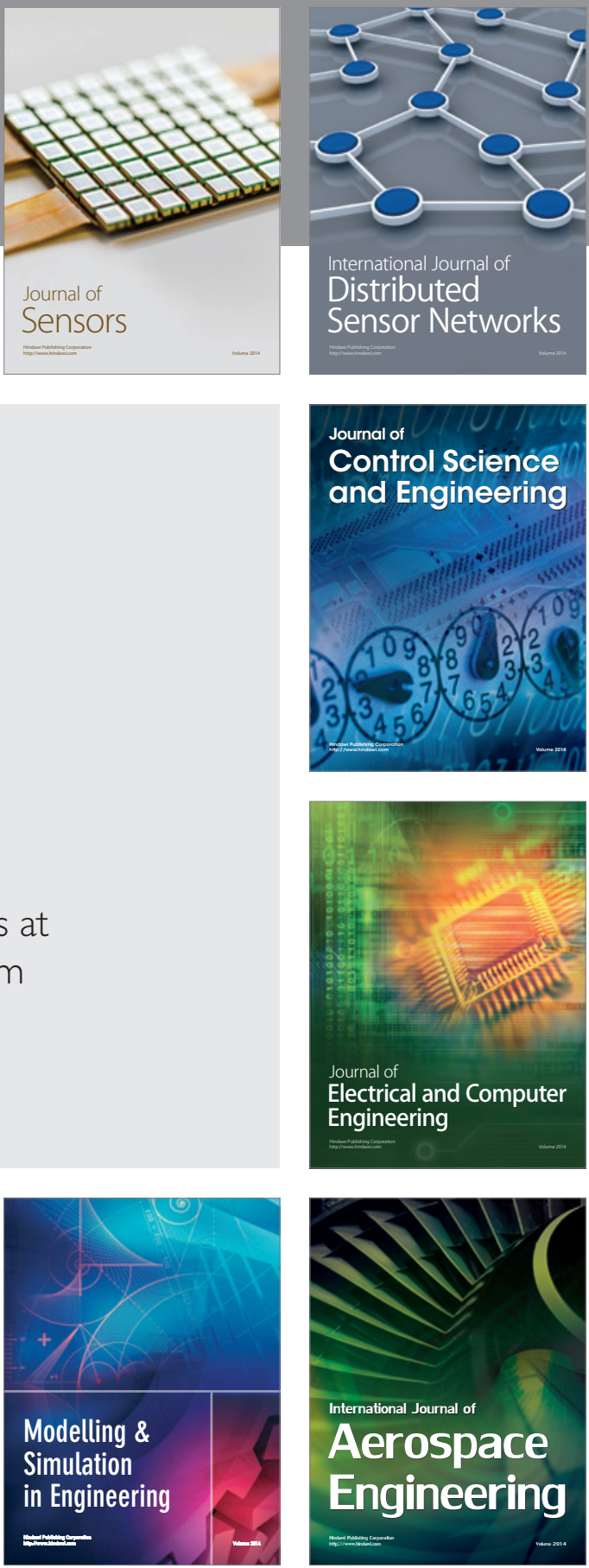

Journal of

Control Science

and Engineering
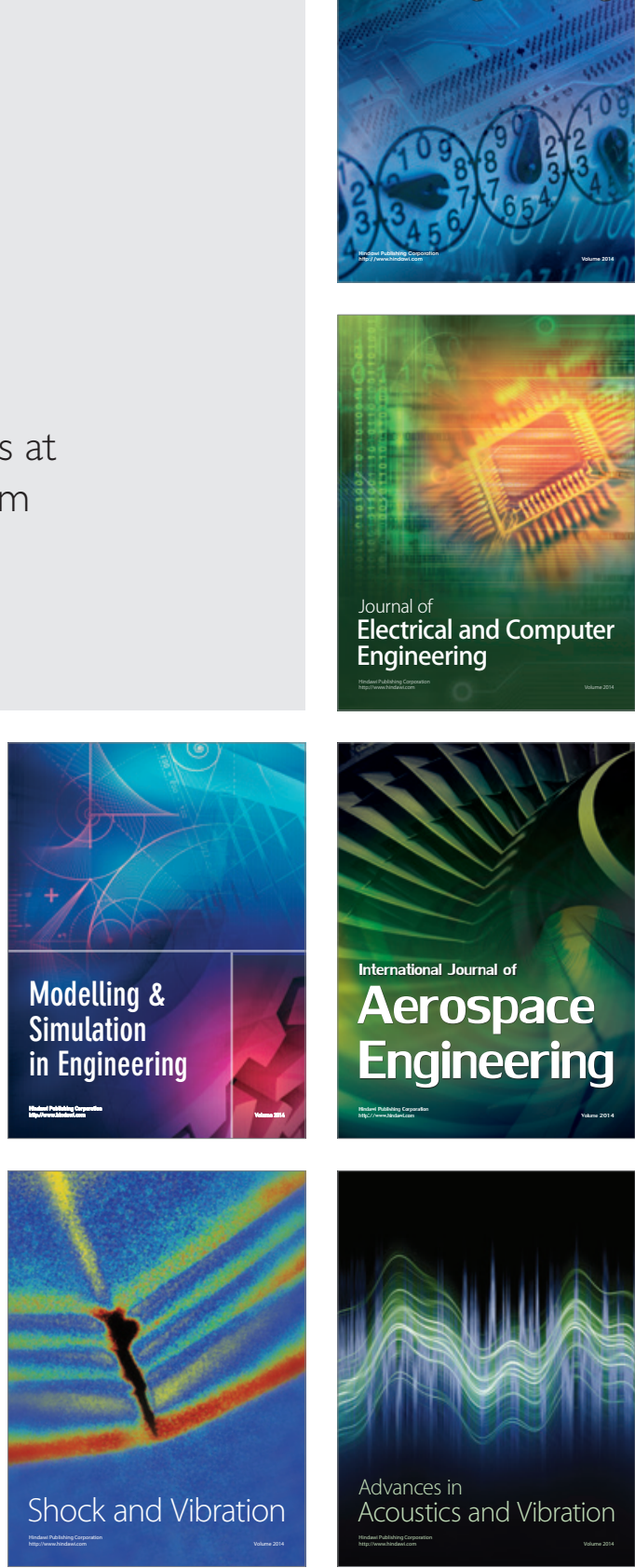